\title{
Older Adults' Persistence to Antihypertensives Prescribed at Hospital Discharge: a Retrospective Cohort Study
}

J Gen Intern Med 36(12):3900-2

DOI: $10.1007 / \mathrm{s} 11606-020-06401-0$

(C) Society of General Internal Medicine 2021

$\mathrm{F}$ ollowing hospitalization, patients are commonly discharged with changes to their outpatient medications, including the intensification of hypertension regimens made in response to elevated inpatient measurements. ${ }^{1}$ Recent studies indicate discharging older adults' with intensified antihypertensives may not reduce subsequent outpatient blood pressures (BPs) or cardiovascular events. ${ }^{2}$ We sought to characterize older adults' persistence to antihypertensives prescribed following hospitalization, hypothesizing that persistence would be lower for intensified antihypertensives compared to continued outpatient antihypertensives.

\section{METHODS}

The underlying study population has been described previously and consisted of adults age $\geq 65$ years with hypertension who were hospitalized in the national Veterans Health Administration (VHA) health system for pneumonia, venous thromboembolism or urinary tract infection, between 2011 and 2013 and followed for 18 months after discharge. ${ }^{1,2}$ VHA pharmacy data was used to assess antihypertensive use and to classify antihypertensives filled at discharge as continuations, dose increases, dose decreases, or new starts.

For each antihypertensive prescribed at discharge, we calculated persistence as the number of days between the discharge fill and the last refill for the same dose or greater, plus the days' supply of the latest refill. ${ }^{3} \mathrm{We}$ report persistence to 12 months following discharge, and to avoid undercounting due to transient nonadherence, we assessed refill history for 18 months. We constructed Kaplan-Meier curves and used log-rank tests to examine the differences in persistence by intensification type. Analyses were conducted using Stata 14.1. This research was approved by the San Francisco Veteran Affairs Medical Center institutional review board.

\section{RESULTS}

The study population included 3839 older adults (mean age 76.5 [SD 8.3], 97.5\% male) who filled 5534 antihypertensives at discharge (Table 1). One-third of patients filled multiple antihypertensives and persistence to each fill was examined independently.

Of the 5534 antihypertensive fills examined, 2041 (36.9\%) were new starts, 695 (12.6\%) dose increases, 2135 (38.6\%) continuations, and 663 (12.0\%) dose decreases. Overall, 2032 (36.7\%) of discharge fills were never refilled and 2924 $(52.8 \%)$ were no longer being filled by 1 year. Persistence was higher for continuations and dose decreases compared to dose increases (Fig. 1) $(P<.001)$. Persistence to new starts was lower than persistence to all other fill types with $44.0 \%$ of discharge fills never refilled and $60.0 \%$ no longer in use at 1 year.

\section{DISCUSSION}

In this study of antihypertensives prescribed to older adults at hospital discharge, one-third of antihypertensives were never refilled and half were not continued at 1 year, with lower persistence to intensified antihypertensives compared to continuations. These findings help explain prior observations that discharge with intensified antihypertensives is not associated with improved outpatient BP control. ${ }^{3}$ 
Table 1 Characteristics of Older Adults Filling Antihypertensive Prescriptions at Hospital Discharge

\begin{tabular}{|c|c|}
\hline Characteristic & $N=3839$ \\
\hline \multicolumn{2}{|l|}{ Demographics } \\
\hline Age, mean (SD), years & $76.5(8.3)$ \\
\hline Male sex, no. (\%) & $3742(97.5)$ \\
\hline \multicolumn{2}{|l|}{ Race/ethnicity, no. (\%) } \\
\hline White & $2761(71.9)$ \\
\hline Black & $858(22.4)$ \\
\hline Hispanic & $96(2.5)$ \\
\hline Other & $24(3.2)$ \\
\hline \multicolumn{2}{|l|}{ Region, no. $(\%)^{\mathrm{b}}$} \\
\hline Northeast (VISN 1-4) & $526(13.7)$ \\
\hline South (VISN 5-9, 16, 17) & $1837(47.9)$ \\
\hline Midwest (VISN 10-12,15, 23) & $773(20.1)$ \\
\hline West (VISN 18-22) & $703(18.3)$ \\
\hline \multicolumn{2}{|l|}{ Hospitalization characteristics } \\
\hline \multicolumn{2}{|l|}{ Reason for hospitalization, No. $(\%)$} \\
\hline Pneumonia & $1982(51.6)$ \\
\hline Urinary tract infection & $1415(36.9)$ \\
\hline Venous thromboembolism & $442(11.5)$ \\
\hline Length of stay, median (IQR), days & $4(3$ to 7$)$ \\
\hline \multicolumn{2}{|l|}{ Comorbidities, no. (\%) } \\
\hline \multicolumn{2}{|l|}{ Charlson Comorbidity score } \\
\hline 0 (good health) & $207(5.4)$ \\
\hline 1-3 (average health) & $1496(39.0)$ \\
\hline$>4$ (poor health) & $2136(55.6)$ \\
\hline \multicolumn{2}{|l|}{ Sēected comorbidities } \\
\hline Prior myocardial infarction & $506(13.2)$ \\
\hline Congestive heart failure & $1281(33.4)$ \\
\hline Cerebrovascular disease & $939(24.5)$ \\
\hline Renal disease & $1494(38.9)$ \\
\hline Diabetes & $1957(51.0)$ \\
\hline Dementia & $268(7.0)$ \\
\hline Metastatic malignancy & $194(5.1)$ \\
\hline \multicolumn{2}{|l|}{ Pre-hospitalization medication use } \\
\hline Medications on admission, median (IQR) & $8(5$ to 11$)$ \\
\hline \multicolumn{2}{|l|}{ Antihypertensives on admission, no (\%) } \\
\hline 0 & $591(15.4)$ \\
\hline 1 & $942(24.5)$ \\
\hline 2 & $1181(30.8)$ \\
\hline 3 & $743(19.4)$ \\
\hline $4+$ & $382(10.0)$ \\
\hline \multicolumn{2}{|l|}{ Discharge antihypertensives fills, no. (\%) } \\
\hline Single antihypertensive filled & $2559(66.7)$ \\
\hline Multiple antihypertensives filled & $1280(33.3)$ \\
\hline \multicolumn{2}{|l|}{ Discharge medication changes } \\
\hline Any intensification ${ }^{\mathrm{a}}$ & $2119(55.2)$ \\
\hline Any new antihypertensive start & $1645(42.8)$ \\
\hline Any dose increase & $640(16.7)$ \\
\hline Any dose decrease & $588(15.3)$ \\
\hline
\end{tabular}

${ }^{a}$ Includes new antihypertensive starts and dose increases

${ }^{b}$ Administrative divisions in the VA are called Veterans Integrated Service Networks (VISNs) which were divide to roughly approximate US Census divisions
Persistence to continued and intensified antihypertensives was poor, suggesting that hospitalization may significantly disrupt outpatient hypertension management plans. These results build on prior research indicating that adherence to antihypertensives prescribed following acute cardiac hospitalizations was similarly low, ${ }^{4}$ indicating that a greater emphasis on chronic disease monitoring and supporting adherence is necessary following discharge, regardless of the primary reason for hospitalization.

Patients may experience adverse drug events or medication confusion leading to discontinuation. Non-persistence to discharge intensified antihypertensives may be appropriate when patients with previously well-controlled outpatient BP are discharged with unnecessary changes due to transiently elevated inpatient BPs. ${ }^{1}$ Furthermore, outpatient clinicians may reverse changes based upon contextual knowledge of patients' prior BP control, likelihood to benefit, or preferences.

This study focused on older adults receiving care in the VHA system, which provides inpatient, outpatient, and pharmacy services with a shared electronic medical record and may not be generalizable to other populations. Reliance on pharmacy fill databases may overestimate medication use for patients with intermittent adherence. We did not assess the reasons for discontinuation of antihypertensives following discharge.

In light of these results and prior studies of discharge prescribing outcomes, ${ }^{1,2,4}$ a reconsideration of perihospitalization hypertension management is warranted. ${ }^{5}$ To improve persistence, efforts to improve chronic disease management across transitions of care should not be limited to medication reconciliation, but must ensure a clear chronic disease care plan is in place, with agreement from the patient and their outpatient clinicians, particularly when outpatient medication regimens are adjusted at discharge. Consideration should be given to minimizing intensification of antihypertensives during unrelated hospitalizations and instead communicating concerns about inpatient BPs to outpatient clinicians for close follow-up and consideration of medication changes following recovery from acute illness.

Acknowledgments: We thank Malena Spar and Anael Rizzo (University of California, San Francisco) for project management assistance. 


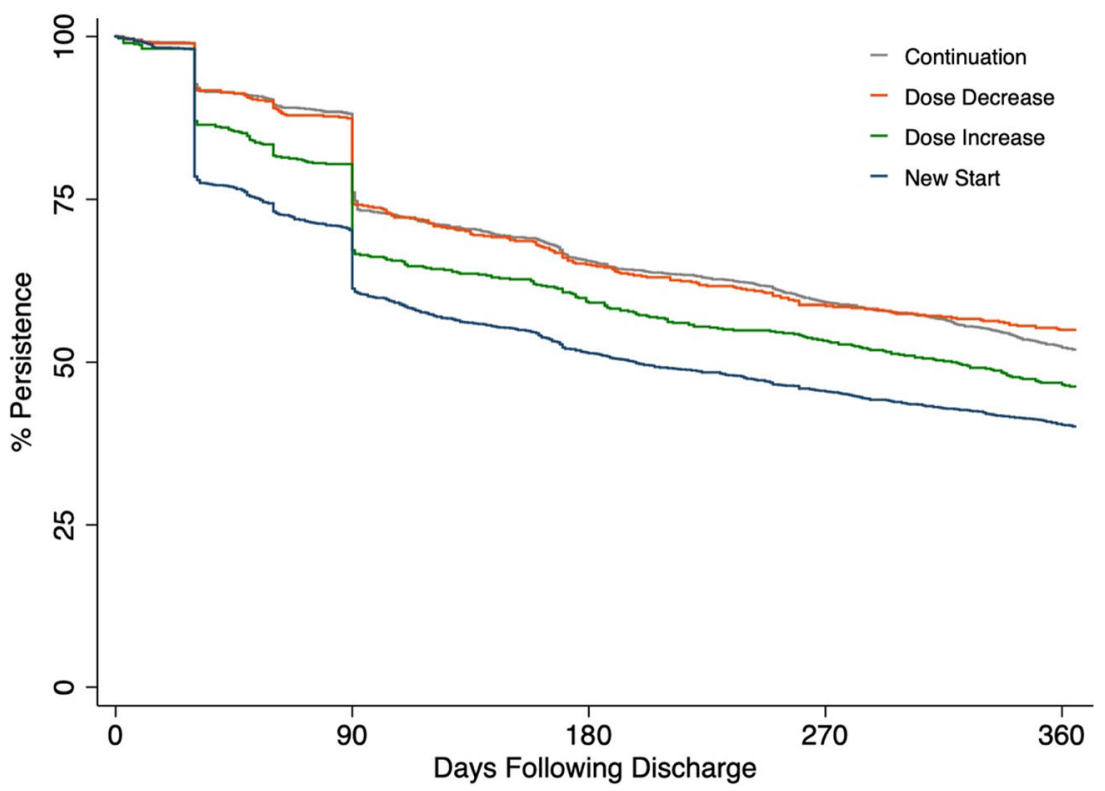

Figure 1 Persistence with antihypertensives intensified at hospital discharge. Note: Survival curves for antihypertensives prescribed at hospital discharge, among older adults 65 years and older with a past diagnosis of hypertension who were discharged from a Veterans Health Administration medical centers following hospitalization for non-cardiovascular medical conditions. Continuations, dose decreases, and dose increases defined as fills of a discharge prescription for an antihypertensive used prior to hospitalization at the total daily same dose $\pm 20 \%$, at $<20 \%$ the pre-hospitalization dose, or at $>\mathbf{2 0 \%}$ the pre-hospitalization dose respectively. New antihypertensive starts defined as fill of a discharge prescription for an antihypertensive not being used prior to hospitalization. Log rank test for the difference between groups $P<.001$.

Timothy S. Anderson, MD, MAS $S^{1,2}$

Bocheng Jing, $M S^{3,4}$

Kathy Fung, $M S^{3,4}$

Michael A. Steinman, $M D^{3,4}$

${ }^{1}$ Division of General Medicine, Beth Israel Deaconess Medical Center,

Boston, MA, USA

${ }^{2}$ Richard A. and Susan F. Smith Center for Outcomes Research in Cardiology, Beth Israel Deaconess Medical Center,

Boston, MA, USA

${ }^{3}$ San Francisco VA Medical Center,

San Francisco, CA, USA

${ }^{4}$ Division of Geriatrics, University of California,

San Francisco, CA, USA

Corresponding Author: Timothy S. Anderson, MD, MAS; Division of General Medicine, Beth Israel Deaconess Medical Center, Boston, MA, USA (e-mail: tsander1@bidmc.harvard.edu).

Author Contributions Drs. Anderson and Steinman had full access to all the data in the study and take responsibility for the integrity of the data and the accuracy of the data analysis.

Study concept and design: Anderson and Steinman

Acquisition, analysis, or interpretation of data: All authors

Drafting of the manuscript: Anderson

Critical revision of the manuscript for important intellectual content: All authors

Statistical analysis: Anderson, Jing, and Fung

Obtained funding: Anderson and Steinman

Administrative, technical, or material support: Steinman

Study supervision: Steinman

Funding Dr. Anderson was supported by grants from the National Institute on Aging (L3OAG060493 and R03AG064373). Dr. Steinman was supported by grants from the National Institute on Aging
(K24AG049057, P30AG044281, and R24AG064025). All other authors report receiving no external funding related to this work.

\section{Compliance with Ethical Standards:}

Conflict of Interest: The authors declare that they do not have a conflict of interest.

Disclaimer: The views expressed herein are those of the authors and do not necessarily represent the views of the US Department of Veterans Affairs or the University of California, San Francisco. The funding organizations had no role in the design and conduct of the study; collection, management, analysis, and interpretation of the data; preparation or approval of the manuscript; and decision to submit the manuscript for publication.

\section{REFERENCES}

1. Anderson TS, Wray CM, Jing B, Fung K, Ngo S, Xu E, Shi Y, Steinman MA. Intensification of older adults' outpatient blood pressure treatment at hospital discharge: national retrospective cohort study. BMJ. 2018;362:k3503.

2. Anderson TS, Jing B, Auerbach A, et al. Clinical Outcomes After Intensifying Antihypertensive Medication Regimens Among Older Adults at Hospital Discharge. JAMA Intern Med. 2019;179(11): 1528-1536.

3. Raebel MA, Schmittdiel J, Karter AJ, Konieczny JL, Steiner JF. Standardizing terminology and definitions of medication adherence and persistence in research employing electronic databases. Med Care. 2013;51(8 Suppl 3):S11-S21.

4. Choudhry NK, Setoguchi S, Levin R, Winkelmayer WC, Shrank WH. Trends in adherence to secondary prevention medications in elderly postmyocardial infarction patients. Pharmacoepidemiol Drug Saf. 2008;17:1189-1196

5. Anderson TS, Wray CM. Annals for Hospitalists Inpatient Notes Inpatient Hypertension-To Treat or Tolerate?. Ann Intern Med. 2020; 172(8):HO2-HO3.

Publisher's Note: Springer Nature remains neutral with regard to jurisdictional claims in published maps and institutional affiliations. 